\title{
Evaluation of the specificity and effectiveness of selected oral hygiene actives in salivary biofilm microcosms

\begin{abstract}
Correspondence
Andrew J. McBain

andrew.mcbain@

manchester.ac.uk
\end{abstract} \\ Received 14 July 2010 \\ Accepted 15 August 2010

\author{
Ruth G. Ledder, ${ }^{1}$ Prem K. Sreenivasan, ${ }^{2}$ William DeVizio ${ }^{2}$ \\ and Andrew J. McBain ${ }^{1}$ \\ ${ }^{1}$ School of Pharmacy and Pharmaceutical Sciences, University of Manchester, \\ Manchester M13 9PL, UK \\ ${ }^{2}$ Colgate-Palmolive Company, 909 River Road, Piscataway, NJ 08855, USA
}

\section{INTRODUCTION}

Regimens for the routine control of dental plaque include the use of formulations that are commonly augmented with active antimicrobial compounds in order to enhance the inactivation and/or removal of adherent bacteria (for a review see Marsh, 1991). Clinically effective agents generally manifest significant inhibitory activity against oral bacteria, possess properties suited to the production of stable formulations and exhibit substantivity (sustained in situ antimicrobial activity) (Elworthy et al., 1996; Marsh, 1992). Compounds that have demonstrated clinical efficacy in controlling dental plaque include metal ions such as stannous fluoride (SF) (Weiland et al., 2008) and zinc lactate (ZL) (Barnes et al., 2008) together with antimicrobials such as triclosan (TCS) (2,4,4'-trichloro-2'-hydroxydiphenyl ether) (Gilbert et al., 2007). SDS, a common ingredient in

Abbreviations: CDFF, constant-depth film fermenter; DGGE, denaturing gradient gel electrophoresis; HA, hydroxyapatite; HDM, hydroxyapatite disc model; MDFR, modified drip-flow biofilm reactor; MSD, multiple Sorbarod device; SF, stannous fluoride; TCS, triclosan; ZLSF, zinc lactate with stannous fluoride in combination. dentifrices, is a foaming agent that also has antibacterial properties (Jenkins et al., 1991). The oral microbiota is a taxonomically diverse bacterial ecosystem and is estimated to contain several hundred phylogenetically distinct species based on 16S rRNA gene analyses (Bik et al., 2010; Paster et al., 2001), less than half of which have been cultured (Paster et al., 2001). Whilst routine bacterial cultivation with variously selective media targets a limited proportion of this diverse population, these include numerically and functionally important bacteria (Paster et al., 2001). Much of the current understanding of the effectiveness of dental antimicrobials has been based on measurable clinical benefits, such as the reduction of caries and the control of gum inflammation, and on the microbiology, in terms of effects on total numbers of cultivable bacteria. In vitro studies have enabled insights to be gained about the comparative effectiveness and specificity of formulations for important functional groups of bacteria, and avoid issues of compliance whilst minimizing variation.

Since antibacterial adjuncts for oral hygiene are generally incorporated into complex formulations (Marsh, 1992) 
and the majority of investigations into their effects on complex plaques either in vivo or in vitro have been done using formulations, the understanding of the contributions of active components would be further enhanced by determining the antibacterial and microbiological effects of the actives in the absence of excipients. The aim of the current investigation, therefore, was to comparatively evaluate the antimicrobial activity and specificity of actives utilized in widely used oral hygiene formulations. These were SDS, SF, TCS, ZL and ZL with SF in combination (ZLSF). Two types of biofilm model were utilized: a hydroxyapatite disc model (HDM) (Ledder et al., 2009) and the modified drip-flow biofilm reactor (MDFR) (Xu et al., 1998). The HDM is amenable to high throughput antimicrobial assays and was utilized to dose immature plaques, whilst the MDFR is a proprietary system that supports biofilms on microscope slides by continuous drip-feeding and thus enables the effects of sustained dosing of mature plaques to be monitored. Both systems provide information about the comparative efficacy and specificity of the actives against various functional groups of oral bacteria: HDMs in short-term experiments and MDFRs generally for longer durations. In the current study, differential viable counting was used to enumerate important functional groups of oral bacteria and in order to confirm the stable maintenance of inoculum diversity in the MDFRs and the effects of the primary active systems; the effects of TCS and ZLSF were also monitored using eubacterial-specific PCR-denaturing gradient gel electrophoresis (DGGE).

\section{METHODS}

Chemicals. Unless otherwise stated, chemicals were obtained from Sigma. Formulated bacteriological media were purchased from Oxoid. Hydroxyapatite (HA) disc and HA-coated slides were obtained from Clarkson Chromatography.

Saliva samples. These were obtained by expectoration into a sterile Universal bottle over the course of $30 \mathrm{~min}$ by two volunteers (1 male, 1 female) who did not have extant dental disease. Collected saliva was used to inoculate models, as outlined below.

HDMs. Individual saliva $(1 \mathrm{ml})$ was dispensed into each of 8 wells of a sterile 24-well tissue culture plate within $20 \mathrm{~min}$ of collection. HA discs ( $9.6 \mathrm{~mm}$ diameter) were sterilized by autoclaving then transferred aseptically into the wells. Inoculated plates were mounted onto an orbital shaker and continuously mixed gently at 144 oscillations $\mathrm{min}^{-1}$ in a mark 3 anaerobic work station (Don Whitley Scientific) (with the following gas mix: $80 \% \mathrm{~N}_{2}, 10 \% \mathrm{CO}_{2}$ and $10 \%$ $\mathrm{H}_{2}$ ) for $12 \mathrm{~h}$ at $37{ }^{\circ} \mathrm{C}$. After this time, antimicrobial agents were added as described below. For enumeration, an HA disc was aseptically removed from the tissue culture plate and processed as outlined below.

Drip-flow biofilm reactors. For multiple exposure experiments, MDFRs (Biosurfaces Technologies) (Xu et al., 1998) were used to generate biofilm microcosms using human saliva as inocula. Each MDFR module delivered a drop-wise, continuous flow of medium over four HA-coated slides $(75 \mathrm{~mm} \times 25 \mathrm{~mm})$ housed in separate parallel channels, each of which measures $102 \mathrm{~mm} \times 30 \mathrm{~mm}$. Each polycarbonate chamber is fitted with an individual removable lid.
During operation, the reactors were placed on feet that allowed an incline to be set at an angle of $10^{\circ}$ from the horizontal to facilitate media flow over the substrata. Before each experimental run the reactors were autoclaved with $\mathrm{HA}$ slides in situ. Temperature $\left(36 \pm 0.5^{\circ} \mathrm{C}\right)$ was maintained by housing the devices within a Perspex incubation chamber (Stuart Scientific). Prior to inoculation, the HA slides were conditioned for $24 \mathrm{~h}$ with culture medium, which was continuously added to each reactor by a peristaltic pump $\left(9.6 \pm 0.2 \mathrm{ml} \mathrm{h}^{-1}\right.$; Watson Marlow). The growth medium comprised a modified artificial saliva medium with cysteine (McBain et al., 2003b). Medium pumps were switched off during inoculation where $500 \mu \mathrm{l}$ fresh saliva was added to each slide. Fresh portions of the inocula were archived at $-60{ }^{\circ} \mathrm{C}$ for subsequent PCR-DGGE analysis and samples of developed microcosm plaques were removed for immediate bacteriological analyses or stored at $-80{ }^{\circ} \mathrm{C}$. For sampling, one slide was removed from each module on days 1, 2, 3 and 6 after dosing commenced.

Addition of antimicrobial agents to microcosms. Antimicrobial agents were prepared in sterile distilled water at concentrations used in dentifrice formulations as follows: TCS suspension $(0.30 \% \mathrm{w} / \mathrm{v})$, SDS $(1.00 \% \mathrm{w} / \mathrm{v})$, ZL $(2.50 \% \mathrm{w} / \mathrm{v})$ and SF $(0.45 \% \mathrm{w} / \mathrm{v})$. Antimicrobials $(500 \mu \mathrm{l})$ (or sterile distilled water for control discs) were delivered to each well of the HDMs $(n=3)$ for $20 \mathrm{~min}$, without removing the tissue culture plate from the shaker and experiments were performed in triplicate using previously reported methods (Ledder et al., 2009). Prior to the addition of antimicrobial agents the MDFRs $(n=4)$ were run for $24 \mathrm{~h}$ after which the reactors received SF, SDS, TCS, ZLSF, ZL or sterile distilled water (control) every 6 h over 6 days by peristaltic pump at a rate of $8 \mathrm{ml} \mathrm{h}^{-1}$ for $10 \mathrm{~min}$.

Differential bacteriological enumeration. HA discs were aseptically removed from the tissue culture plate of the HDMs for enumeration before being gently washed by a single, rapid immersion PBS in order to remove excess medium and dosed components. Samples from the MDFRs were similarly treated and biomass was sampled by scraping and washing as outlined by Goeres et al. (2009). The removed biofilm or disc was immersed in pre-reduced, halfstrength thioglycolate medium (9 ml, United States Pharmacopeia) and vortexed thoroughly for $1 \mathrm{~min}$. Samples were then serially diluted and appropriate dilutions $(0.1 \mathrm{ml})$ were plated in triplicate onto a variety of selective and non-selective media as follows: WikinsChalgren agar (total anaerobes), Wilkins-Chalgren agar with Gramnegative supplements (total Gram-negative anaerobes); trypticase yeast extract, cysteine, sucrose agar (total streptococci) and nutrient agar (total aerobes and facultative anaerobes). Immediately after plating these were placed in an anaerobic chamber (as described above) and maintained at $37{ }^{\circ} \mathrm{C}$ for up to 5 days, except for nutrient agar, which was incubated aerobically for 3 days. Portions of biofilm from the MDFRs were archived at $-60{ }^{\circ} \mathrm{C}$ for subsequent PCR-DGGE analysis.

Eubacterial community profiling using PCR-DGGE. DNA was extracted from the archived MDFR samples using a DNA stool mini kit (Qiagen) in accordance with the manufacturer's instructions for analysis by PCR-DGGE as previously described (McBain et al., $2003 \mathrm{~b}$ ). Resolved bands, representative of unique band positions were excised from the polyacrylamide gels and sequenced for identification using previous published methods (Ledder et al., 2007).

Dendrogram construction for cluster analysis. Gel images were processed using Adobe Photoshop Elements version 7 and analysed using the BioNumerics Fingerprint package (Applied Maths). The bands present in each lane were detected automatically and then checked manually. A reference lane was created using bands present in each lane to generate matching profiles. The matching profiles for each lane were used to produce an UPGMA (Sneath \& Sokal, 1973) dendrogram so that clustering patterns could be determined. 
Statistical analyses. For analyses of viable count data Students $t$-test was used to determine whether the effects of the antimicrobials in the microcosms were significant. For this, data were arranged in tables and subjected to a Microsoft Excel macro. Eubacterial diversity within PCR-DGGE consortial profiles was assessed using the Shannon-Weaver index of diversity $\left(H^{\prime}\right)$ (Ledder et al., 2007; Gafan et al., 2005) according to the following equation:

$H^{\prime}=-\sum_{i=1}^{s} p^{i} \ln \left(p^{i}\right)$

where $s$ is the number of species (species richness) and $p^{i}$ is the proportion of species in the sample $i$. $H^{\prime}$ was calculated for controls over time and also for plaques dosed with TCS and ZLSF. The MannWhitney $U$ test was then performed on selected samples using SPSS version 11.5.

\section{RESULTS AND DISCUSSION}

In the current investigation, differential culture and PCRDGGE were used to monitor ecological changes caused by selected active compounds commonly used in dentifrices within two distinct oral biofilm microcosm systems. Whilst differential isolation is a well-established method for the enumeration of major functional or taxonomic groups of oral bacteria such as the Gram-negative anaerobes and streptococci, PCR-DGGE can be used to (i) produce corroborative data, (ii) assess the temporal stability and compositional concordance of microcosm plaques with progenitor consortia (i.e. salivary inocula) and (iii) facilitate the statistical and comparative assessment of antimicrobial effects of various treatments.

\section{Single dosing of HDM plaques}

HDMs have previously been validated for dosing studies on salivary microcosm biofilms and represent a useful system for concomitantly dosing multiple plaques (Ledder et al., 2009). Fig. 1 shows the effects of a single 20 min exposure to the test compounds on relatively immature plaques grown in HDMs. Following this dosing regime, only SDS and TCS caused significant reductions in bacterial counts, which was manifested for total facultative anaerobes, total anaerobes, and particularly for streptococci and Gramnegative anaerobes, where substantial decreases in viability occurred in comparison to the controls. In contrast, $\mathrm{ZL}$, SF, ZLSF did not significantly decrease counts of any of the taxonomic/functional groups measured in comparison to the controls. Whilst the rapid antibacterial effectiveness associated with TCS and SDS is a desirable property for oral hygiene agents, dentifrices are normally used frequently, and accordingly MDFR plaques were dosed over 6 days in order to compare short and medium-term effects.

\section{Characterization of MDFR plaques}

The MDFR is a proprietary biofilm reactor that uses plain or variously coated microscope slides as substrata for the maintenance of biofilms that are drip-fed with growth

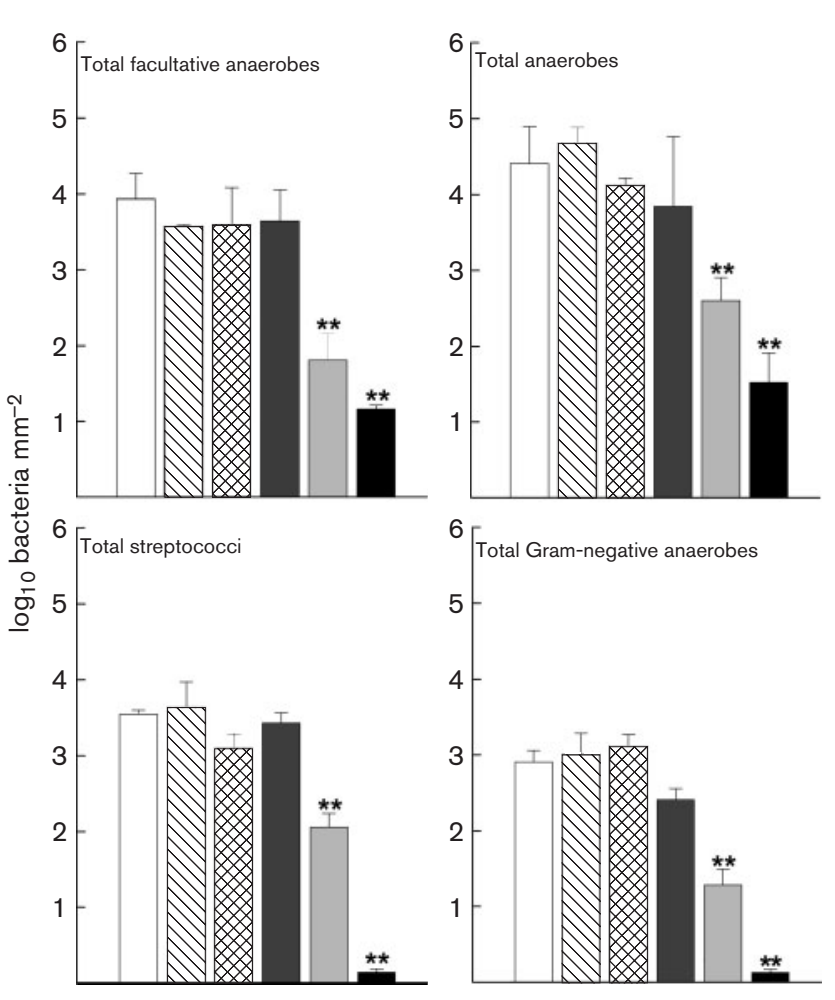

Fig. 1. Effects of actives ZL, SF, ZLSF, SDS and TCS on differential viable bacterial counts in saliva-derived oral microcosms maintained in HDMs after a single 20 min exposure. Data represent distributions for three separate experiments. Error bars indicate SDs. Statistical significance is denoted by * $(P<0.05)$ and ** $(P<0.01)$. White bars, control; hatched bars, ZL; cross-hatched bars, SF; dark grey bars, ZLSF; light grey bars, SDS; black bars, TCS.

medium and may be similarly dosed. The system was developed for the growth of biofilms under slow laminar fluid flow close to the air-liquid interface and has been validated by Goeres et al. (2009) for this purpose using pure cultures of Pseudomonas aeruginosa. It has also been evaluated in comparison to the Centers for Disease Control and Prevention biofilm reactor for use in biofilm disinfectant efficacy tests with axenic cultures of $P$. aeruginosa and Staphylococcus aureus (Buckingham-Meyer et al., 2007). Published reports of the use of MDFRs for dental applications are apparently limited to a brushing study that used pure cultures of the cariogenic oral bacterium Streptococcus mutans (Adams et al., 2002). Therefore, to the best of our knowledge the current investigation is the first to report the use of MDFRs for the continuous culture and antimicrobial treatment of salivary microcosms. HA-coated slides were used as substrata, which was therefore essentially the same material as the discs used in the HDMs. In order to maximize the maintenance of bacterial diversity present in the inoculum, a previously validated mucin-containing growth medium was utilized that had previously supported stable complex 
oral microcosms in constant-depth film fermenters (CDFFs) (McBain et al., 2003a, b; Wong \& Sissons, 2001), HDMs (Ledder et al., 2009) and multiple Sorbarod devices (MSDs) (Ledder et al., 2006, 2009) (a system that grows biofilms on cellulose filters perfused with growth medium; McBain et al., 2005). In order to objectively compare the eubacterial profiles of three randomly selected MDFR microcosms with the salivary inocula from which they developed, dendrograms were constructed from eubacterial-specific PCR-DGGE fingerprints (data not shown). According to these analyses, concordance of microcosms with their corresponding inocula ranged between 58 and $97 \%$, which indicates that a variable proportion of salivary taxa were supported in the model systems during different experimental runs. This variability is greater than data similarly generated with MSDs, where concordance ranged from 70 to $90 \%$ (Ledder et al., 2006; McBain et al., 2005), and may relate to the relatively large surface area of MDFR substrata, together with aerial heterogeneity (McBain, 2009). An additional consideration when assessing the relevance of microcosm model systems for antimicrobial dosing is that steady states should be achieved before dosing. In this respect, the DGGE analyses showed reasonable congruence in control samples over 6 days of continuous culture, which were not significantly different with respect to ecological diversity according to the Mann-Whitney U test (data not shown).

\section{Effects of multiple dosing on MDFR plaques}

The experimental approach adopted in the current study has been outlined in a previous investigation where it was established that individual inocula reproduce much of the inter-individual variation in the salivary eubacterial communities (Ledder et al., 2006). Whilst in vitro dosing regimes do not necessarily precisely reproduce the dynamics and inter-individual variability of human use, the box plots in Figs $2-5$ do give an indication of consortial responses to dosing, similar to those that would occur in situ. Bacteriological data generated after multiple exposures of MDFRs to the test agents (Figs 2-5) show that as with HDM plaques (Fig. 1), TCS was the most consistently effective active tested with respect to the amplitude of viability reductions, and caused significant $(P<0.01)$ reductions for all taxonomic or functional groups [i.e. total facultative anaerobe counts (Fig. 2), total anaerobes (Fig. 3), streptococci (Fig. 4) and Gram-negative anaerobes (Fig. 5)] tested at all time points. The inhibitory activity of TCS against streptococci and Gram-negative anaerobes was particularly marked, and TCS was the only individual active tested that maintained a significant $(P<0.01)$ decrease in the Gram-negative anaerobes throughout the test period in MDFRs (Fig. 5), whilst SDS and ZLSF were also effective, particularly at day 6 (Fig. 5). Since all of the currently known periodontal pathogens are Gram-negative anaerobes (Socransky et al., 1998), this functional group of oral bacteria is a key target group for oral hygiene, and dentifrices containing TCS have previously been reported

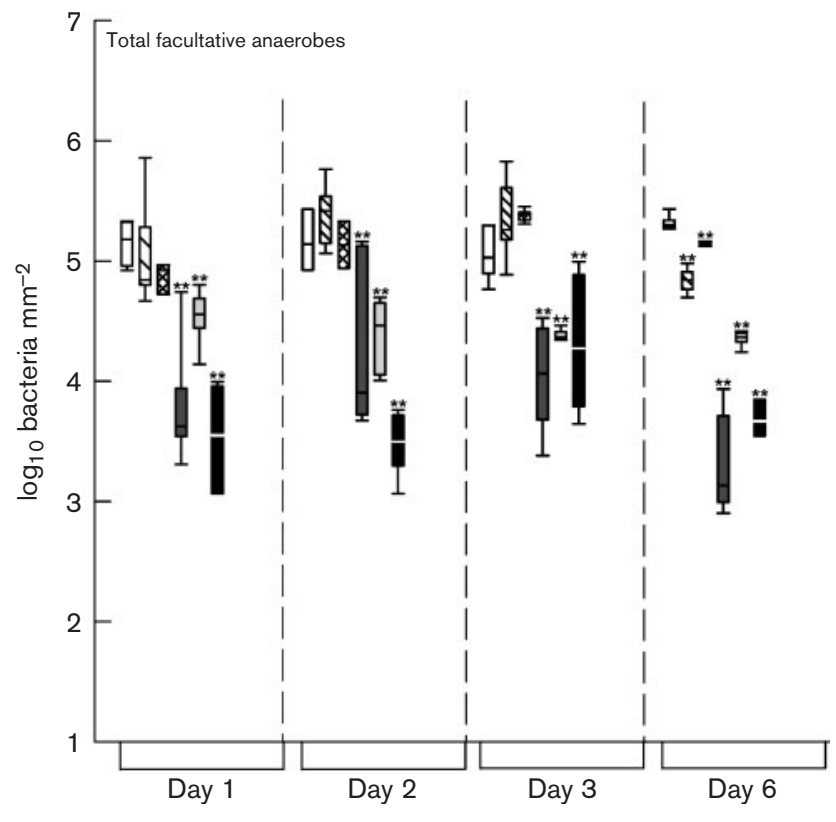

Fig. 2. Effects of actives ZL, SF, ZLSF, SDS and TCS on total viable counts of facultative bacteria during a multiple dosing regime over 6 days in MDFRs. Dosing began on day 1. Data represent distributions for four separate experiments. The horizontal bars within the boxes represent median values; the error bars indicate SDs. Statistical significance is denoted by * $(P<0.05)$ and $^{* \star}(P<0.01)$. See the legend to Fig. 1 for the key.

to significantly reduce gingivitis and periodontitis (Panagakos et al., 2005; Volpe et al., 1996). Other reports concerning the substantial anti-anaerobe effects of TCS and its specificity for Gram-negative anaerobes have most often utilized TCS within complex formulations (Jones et al., 1990; McBain et al., 2003b; Saunders et al., 2000a; Zambon et al., 1990). Saunders and colleagues (Saunders et al., 2000b), however, investigated the ecological effects of TCS and TCS monophosphate using a planktonic, continuous culture microcosm model that simulated caries-like and periodontal disease-like conditions, and reported that Gram-negative anaerobes including Fusobacterium sp. were markedly inhibited in periodontal disease-like microcosms by both forms of TCS.

In contrast to single dosing (Fig. 1), repeated dosing with ZLSF, ZL and SF caused significant reductions in bacterial viability. For ZLSF these were significant $(P<0.01)$ for all test groups of bacteria (Figs 2-5). Whilst data ranges indicate considerable run-to-run variation, mean bacterial reductions caused by ZLSF were generally less marked than those for TCS for the majority of bacterial groups and time points, apart from facultative anaerobes for which mean values were lowered more by ZLSF at days 3 and 6 (Fig. 2). Whilst SF caused a significant, but shortterm reduction in streptococci on day 1 and 2 (Fig. 4), bacterial reductions caused by $\mathrm{ZL}$ and SF individually 


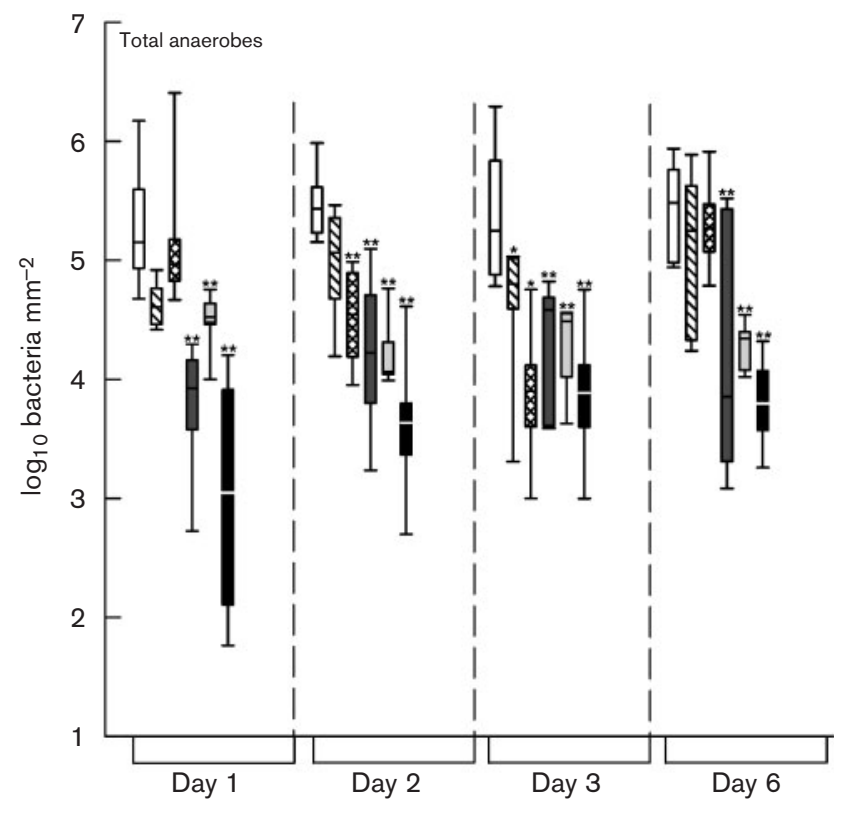

Fig. 3. Effects of actives ZL, SF, ZLSF, SDS and TCS on total viable counts of anaerobic bacteria during a multiple dosing regime over 6 days in MDFRs. See the legend to Fig. 1 for the key.

were inconsistent: being less marked than for the other actives and variable with time. Overall, therefore, $\mathrm{ZL}$ and $\mathrm{SF}$ were the least effective compounds tested with respect

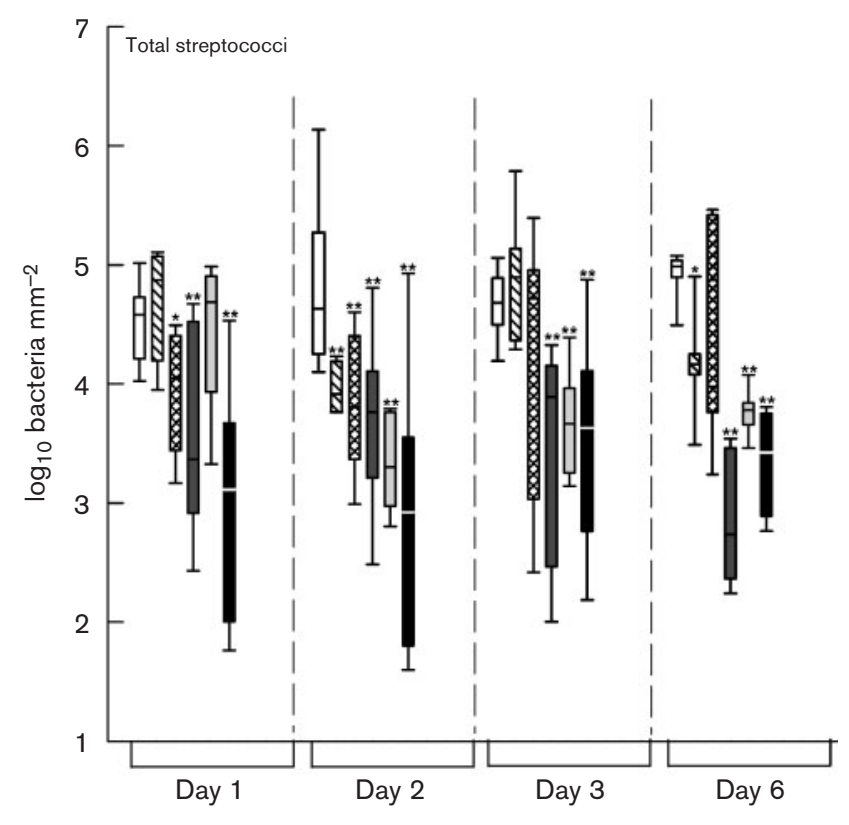

Fig. 4. Effects of actives ZL, SF, ZLSF, SDS and TCS on total viable counts of streptococci during a multiple dosing regime over 6 days in MDFRs. See the legend to Fig. 1 for the key.

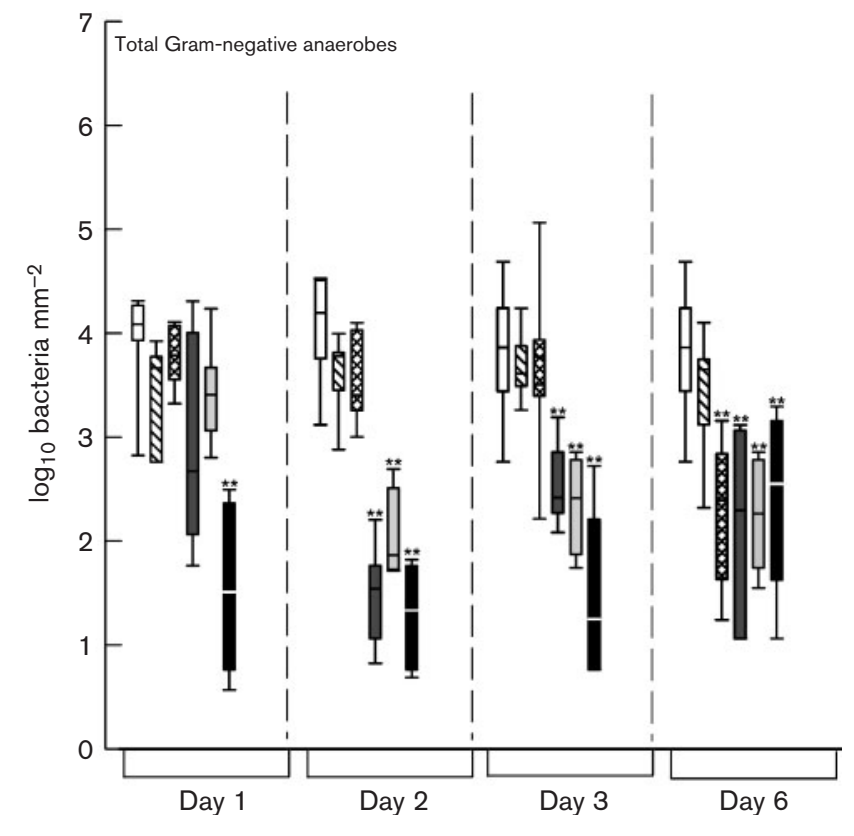

Fig. 5. Effects of actives ZL, SF, ZLSF, SDS and TCS on total viable counts of Gram-negative anaerobes during a multiple dosing regime over 6 days in MDFRs. See the legend to Fig. 1 for the key.

to antibacterial and antiplaque effects. Formulations incorporating SF have been previously reported to be cariostatic, an effect that is reportedly achieved directly by means of enhanced enamel remineralization, by inhibiting the production of acid by oral streptococci (Rolla \& Ellingsen, 1994; Svanberg \& Rolla, 1982) and also presumably through direct antibacterial effects (Addy et al., 1997). Data generated in the current investigation suggest that any antibacterial effects of SF might be manifested more strongly in the oral streptococci than in other functional groups of bacteria, e.g. the Gramnegative anaerobes, and are therefore in agreement with published observations for SF formulations. This effect was not maintained throughout the test period, suggesting that optimal antimicrobial activities of ZL and SF would require multiple dosing and that SF may exhibit reduced effectiveness against mature plaques. Marked antibacterial effects for ZL and SF were not, however, replicated in the HDMs (Fig. 1) either alone or in combination. The anticaries properties of the zinc ion have been attributed to its ability to increase the $\mathrm{pH}$ of dental plaque by inhibiting the glycolytic pathway (He et al., 2002). Previous studies have shown that when zinc and fluoride are used in combination they exert a synergistic effect on the inhibition of glycolysis (Izaguirre-Fernández et al., 1989). Additivity between the tin ion and fluoride may explain the bactericidal effects of ZLSF observed in all functional groups of bacteria studied in this experiment compared to either compound individually (Figs 1-5). 


\section{Consortial profiling with eubacterial-specific PCR-DGGE}

PCR-DGGE enables ecological shifts to be monitored visually, revealing changes in overall diversity that would be difficult to ascertain if analysis were restricted to counting colonies on a limited number of differential agars. It also provides the opportunity to monitor taxa that might not be readily amenable to culture. Both PCR-DGGE and differential viable counting have inherent strengths and weaknesses; culture is biased towards organisms best able to form colonies on agar surfaces whilst PCR-DGGE detects bands that are most amenable to amplification in the competitive PCRs associated with consortial templates. Representative eubacterial profiles generated by PCRDGGE (data not shown) indicate that plaques were stable (i.e. did not significantly vary over time) in terms of total diversity indexes in the absence of dosing (Shannon Weaver index for control plaques was 3.46 (day 1), 3.26 (day 2), 3.33 (day 3 ) and 3.15 (day 6). Since TCS and ZLSF represent ingredients that are used in dentifrice formulations, their effects on MDFR plaques were additionally assessed by PCR-DGGE. Profiles indicated that both ingredients caused major reductions in bacterial diversity that became statistically significant $(P<0.05)$ after 6 days, according to the Mann-Whitney $U$ test. Putative identities of major PCR amplicons indicated that MDFR plaques were dominated by facultative anaerobes, e.g. Actinomyces sp., Citrobacter freundii and Streptococcus sp., together with aerobic species such as Neisseria sp. PCR-DGGE analyses did not reveal a preponderance of strict anaerobes, which, whilst present in the sequences and as indicated by differential bacterial counting, may not have been numerically dominant (data not shown). This contrasts with previous studies using CDFFs (McBain et al., 2003b) and MSDs (McBain et al., 2005), and may be explainable based on the design of the model whereby plaques develop on a flat, open surface. In CDFFs, substrata are commonly set to about $200 \mu \mathrm{m}$ depths and MDS utilize $20 \mathrm{~mm}$ long cellulose filters. This may accelerate the development of the reduced conditions required for the maintenance of anaerobic bacteria within otherwise aerobic environments (Bradshaw et al., 1996; McBain, 2009). It could be argued, therefore, that unless operated in an anaerobic chamber, MDFRs are most suited to modelling supragingival plaque, being largely aerobic and relying on aggregative protection and oxygen gradients to provide anaerobic microenvironments (Bradshaw et al., 1998).

\section{Summary}

Data generated in this investigation have demonstrated that the hierarchy of activity of the tested actives against $e x$ situ plaques was as follows: TCS $>$ SDS $>$ ZLSF $>$ SF $>$ ZL. TCS caused significant reductions in numbers of all groups of bacteria analysed by culture after both single and multiple treatments in complex eubacterial communities. ZLSF also caused significant reductions in numbers of bacteria recovered by culture, but only after multiple dosing. These effects were only infrequently replicated when SF and ZL were dosed individually in MDFRs. Whilst SF was relatively ineffective, it caused significant but short-term reductions in the oral streptococci in MDFR experiments. ZL and SF individually were ineffective following a single 20 min dosing in HDMs, suggesting that these antimicrobials may require repeated dosing in order to accumulate effective levels within plaques or to exert biologically significant effects in target bacteria. PCR-DGGE analyses of MDFR plaques indicated that: (i) the concordance of MDFR eubacterial profiles to saliva inocula ranged between 58 and $97 \%$, (ii) both TCS and ZLSF caused a reduction in bacterial diversity and (iii) the specificities and comparative efficiencies of the actives tested were broadly congruent with those of previous reports.

\section{ACKNOWLEDGEMENTS}

A. J.M. is grateful to Tejal Madhwani and Leon Aarons for constructive comments. This work was funded by Colgate-Palmolive.

\section{REFERENCES}

Adams, H., Winston, M. T., Heersink, J., Buckingham-Meyer, K. A., Costerton, J. W. \& Stoodley, P. (2002). Development of a laboratory model to assess the removal of biofilm from interproximal spaces by powered tooth brushing. Am J Dent 15, 12B-17B.

Addy, M., Greenman, J., Renton-Harper, P., Newcombe, R. \& Doherty, F. (1997). Studies on stannous fluoride toothpaste and gel (2). Effects on salivary bacterial counts and plaque regrowth in vivo. J Clin Periodontol 24, 86-91.

Barnes, V. M., Richter, R., Bastin, D., Lambert, P. \& Xu, T. (2008). Dental plaque control effect of a zinc citrate dentifrice. J Clin Dent 19, 127-130.

Bik, E. M., Long, C. D., Armitage, G. C., Loomer, P., Emerson, J., Mongodin, E. F., Nelson, K. E., Gill, S. R., Fraser-Liggett, C. M. \& Relman, D. A. (2010). Bacterial diversity in the oral cavity of 10 healthy individuals. ISME J 4, 962-974.

Bradshaw, D. J., Marsh, P. D., Allison, C. \& Schilling, K. M. (1996). Effect of oxygen, inoculum composition and flow rate on development of mixed-culture oral biofilms. Microbiology 142, 623629.

Bradshaw, D. J., Marsh, P. D., Watson, G. K. \& Allison, C. (1998). Role of Fusobacterium nucleatum and coaggregation in anaerobe survival in planktonic and biofilm oral microbial communities during aeration. Infect Immun 66, 4729-4732.

Buckingham-Meyer, K., Goeres, D. M. \& Hamilton, M. A. (2007). Comparative evaluation of biofilm disinfectant efficacy tests. J Microbiol Methods 70, 236-244.

Elworthy, A., Greenman, J., Doherty, F. M., Newcombe, R. G. \& Addy, M. (1996). The substantivity of a number of oral hygiene products determined by the duration of effects on salivary bacteria. J Periodontol 67, 572-576.

Gafan, G. P., Lucas, V. S., Roberts, G. J., Petrie, A., Wilson, M. \& Spratt, D. A. (2005). Statistical analyses of complex denaturing gradient gel electrophoresis profiles. J Clin Microbiol 43, 39713978. 
Gilbert, P., McBain, A. \& Sreenivasan, P. (2007). Common therapeutic approaches for the control of oral biofilms: microbiological safety and efficacy. Clin Microbiol Infect 13, 17-24.

Goeres, D. M., Hamilton, M. A., Beck, N. A., Buckingham-Meyer, K., Hilyard, J. D., Loetterle, L. R., Lorenz, L. A., Walker, D. K. \& Stewart, P. S. (2009). A method for growing a biofilm under low shear at the air-liquid interface using the drip flow biofilm reactor. Nat Protoc 4, 783-788.

He, G., Pearce, E. I. \& Sissons, C. H. (2002). Inhibitory effect of $\mathrm{ZnCl}_{2}$ on glycolysis in human oral microbes. Arch Oral Biol 47, 117129.

Izaguirre-Fernández, E. J., Eisenberg, A. D. \& Curzon, M. E. (1989). Interactions of zinc with fluoride on growth, glycolysis and survival of Streptococcus mutans GS-5. Caries Res 23, 18-25.

Jenkins, S., Addy, M. \& Newcombe, R. (1991). Triclosan and sodium lauryl sulphate mouthwashes (I). Effects on salivary bacterial counts. J Clin Periodontol 18, 140-144.

Jones, C. L., Saxton, C. A. \& Ritchie, J. A. (1990). Microbiological and clinical effects of a dentifrice containing zinc citrate and triclosan in the human experimental gingivitis model. J Clin Periodontol 17, 570574 .

Ledder, R. G., Gilbert, P., Pluen, A., Sreenivasan, P. K., De Vizio, W. \& McBain, A. J. (2006). Individual microflora beget unique oral microcosms. J Appl Microbiol 100, 1123-1131.

Ledder, R. G., Gilbert, P., Huws, S. A., Aarons, L., Ashley, M. P., Hull, P. S. \& McBain, A. J. (2007). Molecular analysis of the subgingival microbiota in health and disease. Appl Environ Microbiol 73, 516-523.

Ledder, R. G., Madhwani, T., Sreenivasan, P. K., De Vizio, W. \& McBain, A. J. (2009). An in vitro evaluation of hydrolytic enzymes as dental plaque control agents. J Med Microbiol 58, 482-491.

Marsh, P. D. (1991). Dentifrices containing new agents for the control of plaque and gingivitis: microbiological aspects. J Clin Periodontol 18, 462-467.

Marsh, P. D. (1992). Microbiological aspects of the chemical control of plaque and gingivitis. J Dent Res 71, 1431-1438.

McBain, A. J. (2009). In vitro biofilm models: an overview. Adv Appl Microbiol 69, 99-132.

McBain, A. J., Bartolo, R. G., Catrenich, C. E., Charbonneau, D., Ledder, R. G. \& Gilbert, P. (2003a). Effects of a chlorhexidine gluconate-containing mouthwash on the vitality and antimicrobial susceptibility of in vitro oral bacterial ecosystems. Appl Environ Microbiol 69, 4770-4776.

McBain, A. J., Bartolo, R. G., Catrenich, C. E., Charbonneau, D., Ledder, R. G. \& Gilbert, P. (2003b). Effects of triclosan-containing rinse on the dynamics and antimicrobial susceptibility of in vitro plaque ecosystems. Antimicrob Agents Chemother 47, 3531-3538.
McBain, A. J., Sissons, C., Ledder, R. G., Sreenivasan, P. K., De Vizio, W. \& Gilbert, P. (2005). Development and characterization of a simple perfused oral microcosm. J Appl Microbiol 98, 624-634.

Panagakos, F. S., Volpe, A. R., Petrone, M. E., DeVizio, W., Davies, R. M. \& Proskin, H. M. (2005). Advanced oral antibacterial/antiinflammatory technology: a comprehensive review of the clinical benefits of a triclosan/copolymer/fluoride dentifrice. J Clin Dent 16, S1-S19.

Paster, B. J., Boches, S. K., Galvin, J. L., Ericson, R. E., Lau, C. N., Levanos, V. A., Sahasrabudhe, A. \& Dewhirst, F. E. (2001). Bacterial diversity in human subgingival plaque. J Bacteriol 183, 3770-3783.

Rolla, G. \& Ellingsen, J. E. (1994). Clinical effects and possible mechanisms of action of stannous fluoride. Int Dent J 44, 99-105.

Saunders, K. A., Greenman, J. \& McKenzie, C. (2000a). Ecological effects of triclosan and triclosan monophosphate on defined mixed cultures of oral species grown in continuous culture. J Antimicrob Chemother 45, 447-452.

Saunders, K. A., Greenman, J. \& McKenzie, C. (2000b). Ecological effects of triclosan and triclosan monophosphate on defined mixed cultures of oral species grown in continuous culture. J Antimicrob Chemother 45, 447-452.

Sneath, P. H. A. \& Sokal, R. R. (1973). Numerical Taxonomy. San Francisco, CA: Freeman.

Socransky, S. S., Haffajee, A. D., Cugini, M. A., Smith, C. \& Kent, R. L., Jr (1998). Microbial complexes in subgingival plaque. J Clin Periodontol 25, 134-144.

Svanberg, M. \& Rolla, G. (1982). Streptococcus mutans in plaque and saliva after mouthrinsing with SnF2. Scand J Dent Res 90, 292-298.

Volpe, A. R., Petrone, M. E., De Vizio, W., Davies, R. M. \& Proskin, H. M. (1996). A review of plaque, gingivitis, calculus and caries clinical efficacy studies with a fluoride dentifrice containing triclosan and PVM/MA copolymer. J Clin Dent 7, S1-S14.

Weiland, B., Netuschil, L., Hoffmann, T. \& Lorenz, K. (2008). Substantivity of amine fluoride/stannous fluoride following different modes of application: a randomized, investigator-blind, placebocontrolled trial. Acta Odontol Scand 66, 307-313.

Wong, L. \& Sissons, C. (2001). A comparison of human dental plaque microcosm biofilms grown in an undefined medium and a chemically defined artificial saliva. Arch Oral Biol 46, 477-486.

Xu, K. D., Stewart, P. S., Xia, F., Huang, C. T. \& McFeters, G. A. (1998). Spatial physiological heterogeneity in Pseudomonas aeruginosa biofilm is determined by oxygen availability. Appl Environ Microbiol 64, 4035-4039.

Zambon, J. J., Reynolds, H. S. \& Genco, R. J. (1990). Studies of the subgingival microflora in patients with acquired immunodeficiency syndrome. J Periodontol 61, 699-704. 